\title{
An extraction of jet transport coefficient in cold nuclear matter from world data
}

\author{
Peng Ru, ${ }^{a, *}$ Zhong-Bo Kang, ${ }^{b, c}$ Enke Wang, ${ }^{a}$ Hongxi Xing ${ }^{a}$ and Ben-Wei Zhang ${ }^{d, a}$ \\ ${ }^{a}$ Guangdong Provincial Key Laboratory of Nuclear Science, Institute of Quantum Matter, South China \\ Normal University, Guangzhou 510006, China \\ ${ }^{b}$ Department of Physics and Astronomy, University of California, Los Angeles, California 90095, USA \\ ${ }^{c}$ Mani L. Bhaumik Institute for Theoretical Physics, University of California, Los Angeles, California 90095, \\ USA \\ ${ }^{d}$ Key Laboratory of Quark \& Lepton Physics (MOE) and Institute of Particle Physics, Central China Normal \\ University, Wuhan 430079, China
}

E-mail: p.ru@m.scnu.edu.cn, hxing@m.scnu.edu.cn

\begin{abstract}
Within the theoretical framework of the generalized factorization of perturbative QCD, i.e., the higher-twist expansion formalism, we perform the first global analysis of the jet transport coefficient $(\hat{q})$ in cold nuclear matter, in which analysis we take into account the available data on various types of transverse momentum broadening in $e \mathrm{~A}$ and $p \mathrm{~A}$ collisions, as well as the nuclear modification of the structure functions in DIS. The global analysis suggests a universal and kinematics-dependent $\hat{q}=\hat{q}\left(x, Q^{2}\right)$ in cold nuclear matter, which would be instructive for understanding the jet quenching phenomena and the property of quark-gluon plasma in a more accurate way. Further more, utilizing the Hessian matrix, we evaluate the uncertainty of the extracted $\hat{q}\left(x, Q^{2}\right)$ under the constraints of the current data and generate the error evaluation sets of $\hat{q}\left(x, Q^{2}\right)$, with which we make preliminary predictions for the future EIC experiments at three facilities covering a wide kinematic range.
\end{abstract}

HardProbes 2020

1-6 June 2020

Austin, Texas

\footnotetext{
${ }^{*}$ Speaker
} 


\section{Introduction}

Quantifying the jet transport coefficient $\hat{q}$, which characterizes the strength of the jet-medium interaction, is an important avenue to access the non-perturbative property of the nuclear medium. While $\hat{q}$ is usually simplified as a constant input in the study of the nuclear modification of jet production, theoretical studies [1-4] have indicated possible kinematic (e.g., the jet energy and probing scale) dependence of $\hat{q}$, which has begun to draw increasingly more attentions recently [5-8]. The medium modification of the jet traversing the cold nucleus in electron-nucleus $(e \mathrm{~A})$ and proton-nucleus $(p \mathrm{~A})$ collisions provides a clean observable to test the theoretical framework and to study the kinematic dependence of $\hat{q}$ delicately. In particular, the future EIC experiments [9] are expected to significantly promote the understanding of the jet transport property in cold nuclear matter. In this proceeding, we review our recent study of the $\hat{q}$ in cold nuclear matter via a global analysis of the available world data [5], and present some new results on evaluating the uncertainty of $\hat{q}$ and preliminary predictions for the future EIC experiments.

\section{Universal kinematics-dependent $\hat{q}$ from global analysis: $\hat{q}\left(x_{B}, Q^{2}\right)$}

To theoretically describe the parton multiple scattering in nuclear medium, we utilize the higher-twist (HT) factorization formalism in our global analysis [5], which is a reliable framework extensively used in both hot/dense medium and cold nuclear matter. The experimental data on various types of transverse momentum broadening that are most directly relevant to $\hat{q}$ constitute the backbone of the data sets used in the analysis (listed in Table 1). Take semi-inclusive $e$ A deeply inelastic scattering (SIDIS) for example, the transverse momentum broadening of the final-state hadron, $\Delta\left\langle p_{T}^{2}\right\rangle \equiv\left\langle p_{T}^{2}\right\rangle_{e A}-\left\langle p_{T}^{2}\right\rangle_{e p}$, can be expressed with the universal nonperturbative twist-4 quark-gluon correlation function $T_{q g}\left(x, 0,0, \mu^{2}\right)$, which can be converted into $\hat{q}$ as $T_{q g}\left(x, 0,0, \mu^{2}\right) \approx \frac{9 R_{A}}{8 \pi^{2} \alpha_{s}} f_{q / A}\left(x, \mu^{2}\right) \hat{q}\left(x, \mu^{2}\right)$, with $R_{A}$ the radius of nucleus, $f_{q / A}$ the nuclear PDFs and $\hat{q}$ representing the transverse momentum broadening per unit propagation length of the jet traversing the medium.

In general, $\hat{q}$ would be universal and depend on both the Bjorken $x$ and the probing scale. To consider the kinematic dependence of the non-perturbative $\hat{q}$, we use a parametrization, i.e., $\hat{q}\left(x_{B}, Q^{2}\right)=\hat{q}_{0} \alpha_{s}\left(Q^{2}\right) x_{B}^{\alpha}\left(1-x_{B}\right)^{\beta}\left[\ln \left(Q^{2} / Q_{0}^{2}\right)\right]^{\gamma}$, motivated by the small- $x$ saturation physics, large- $x$ power corrections and the scale dependence from radiative corrections. Table 1 demonstrates

\begin{tabular}{|c|c|c|c|c|c|}
\hline $\begin{array}{l}\text { experimental } \\
\text { data sets (data points) }\end{array}$ & $\begin{array}{r}\chi^{2} \\
\left(\hat{q}_{0}\right)\end{array}$ & $\begin{array}{c}\chi^{2} \\
{\left[\hat{q}\left(x_{B}, Q^{2}\right)\right]} \\
\end{array}$ & $\begin{array}{l}\text { experimental } \\
\text { data sets (data points) }\end{array}$ & $\begin{array}{c}\chi^{2} \\
\left(\hat{q}_{0}\right)\end{array}$ & $\begin{array}{c}\chi^{2} \\
{\left[\hat{q}\left(x_{B}, Q^{2}\right)\right]}\end{array}$ \\
\hline HERMES / SIDIS: $\Delta\left\langle p_{T}^{2}\right\rangle(156)$ & 218.5 & 189.7 & FNAL-E866 / $J / \psi: \Delta\left\langle p_{T}^{2}\right\rangle$ & 2.03 & 2.45 \\
\hline FNAL-E772 / DY: $\Delta\left\langle p_{T}^{2}\right\rangle$ & 2.69 & 1.65 & RHIC $/ J / \psi: \Delta\left\langle p_{T}^{2}\right\rangle$ & 44.4 & 31.0 \\
\hline SPS-NA10 / DY: $\quad \Delta\left\langle p_{T}^{2}\right\rangle$ & 6.86 & 6.47 & $\mathrm{LHC} / J / \psi: \Delta\left\langle p_{T}^{2}\right\rangle$ & 87.3 & 4.8 \\
\hline FNAL-E772 / Y: $\Delta\left\langle p_{T}^{2}\right\rangle$ & 2.33 & 2.67 & FNAL-E665 / DIS: $F_{2}^{A} / F_{2}^{D}(20)$ & 23.7 & 21.46 \\
\hline TOTAL: & 387.9 & 260.2 & & & \\
\hline
\end{tabular}

Table 1: Data sets used in global analysis, and $\chi^{2}$ with constant $\hat{q}=\hat{q}_{0}$ and kinematics dependent $\hat{q}\left(x_{B}, Q^{2}\right)$, respectively. Shown values of $\chi^{2}$ correspond to minimum total $\chi^{2}$ in global analysis [5]. 

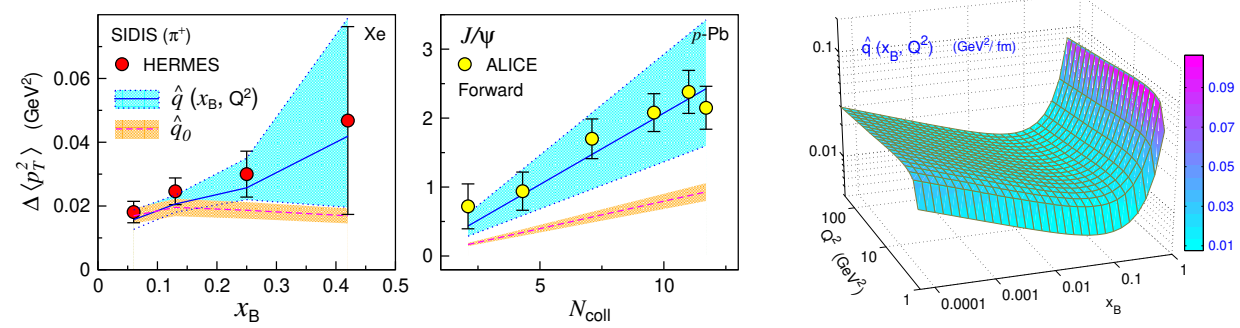

Figure 1: Comparison between $\Delta\left\langle p_{T}^{2}\right\rangle$ calculated with constant $\hat{q}=\hat{q}_{0}$ and kinematics-dependent $\hat{q}\left(x_{B}, Q^{2}\right)$ extracted from global analysis, shown in left (for SIDIS) and middle (for $J / \psi$ ) panels. Shaded areas denote uncertainties from $\hat{q}$ corresponding to $90 \%$ confidence level(C. L.). Right panel shows extracted optimal values of $\hat{q}\left(x_{B}, Q^{2}\right)$.

the total $\chi^{2}$ value obtained in the global analysis with $\hat{q}=\hat{q}\left(x_{B}, Q^{2}\right)$ is significantly smaller than that with constant $\hat{q}=\hat{q}_{0}$. In particular, the theoretical result for $J / \psi$ production at the LHC (related to small- $x$ region) with the constant $\hat{q}$ is far from reasonable. Figure 1 shows that the data on SIDIS and $J / \psi$ production at the LHC can be obviously better reproduced with the consideration of the kinematic dependence of $\hat{q}$. The extracted optimal values of $\hat{q}\left(x_{B}, Q^{2}\right)$ are shown in the right panel of Fig. 1, with which the HT calculations can nicely describe the various data simultaneously [5].

\section{Uncertainty of $\hat{q}$ with Hessian matrix and predictions for future EIC experiments}

To quantify the constraining power of the available data for the kinematics-dependent $\hat{q}$ and to make predictions for future experiments (e.g., EIC), it is very useful to evaluate the uncertainty of the $\hat{q}\left(x_{B}, Q^{2}\right)$ under the constraints of the current data. In Ref. [5], we have used the Lagrange multiplier (LM) method [10] to estimate the uncertainties of the theoretical results of observable. Although the LM method is quite robust, it is very time-consuming for evaluating the uncertainty of $\hat{q}\left(x_{B}, Q^{2}\right)$ and for producing any predictions for future measurements. Recently, we have produced four error evaluation sets $\left(S_{ \pm k}, k=1, \ldots 4\right)$ of $\hat{q}\left(x_{B}, Q^{2}\right)$ utilizing the Hessian matrix method [10], with which we extract the uncertainty of $\hat{q}\left(x_{B}, Q^{2}\right)$ as shown in Fig. 2. The uncertainties are considerable at small- $x$ and are largest at large- $x$, due to the rare constraints from the current data, which are expected to be further constrained by the future measurements. In panels (a) and (b) of Fig. 3, we show the uncertainties of the theoretical calculations with Hessian and LM methods. The good agreement between the two methods demonstrates the reliability of the Hessian method.
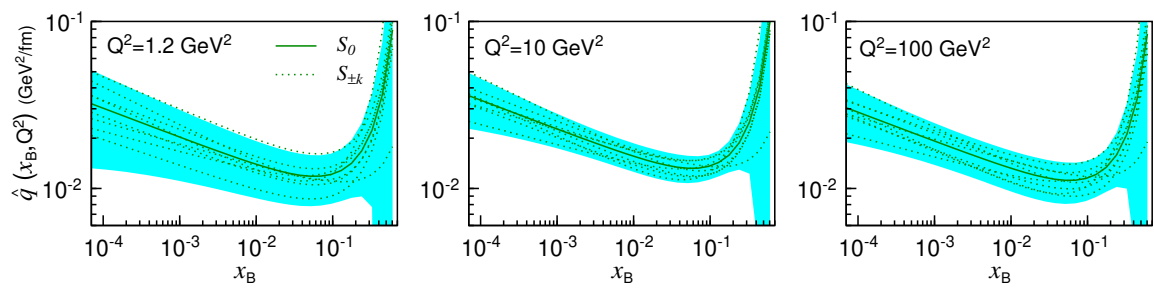

Figure 2: Extracted $\hat{q}\left(x_{B}, Q^{2}\right)$ and its uncertainty. Solid curve represents optimal values $\left(S_{0}\right)$ corresponding to minimum global $\chi^{2}$. Dotted curve represents error evaluation set $\left(S_{ \pm k}, k=1, \ldots 4\right)$ of $\hat{q}\left(x_{B}, Q^{2}\right)$ obtained with Hessian matrix corresponding to $90 \%$ C. L.. Shaded area denotes uncertainty of $\hat{q}$ estimated with $S_{ \pm k}$. 

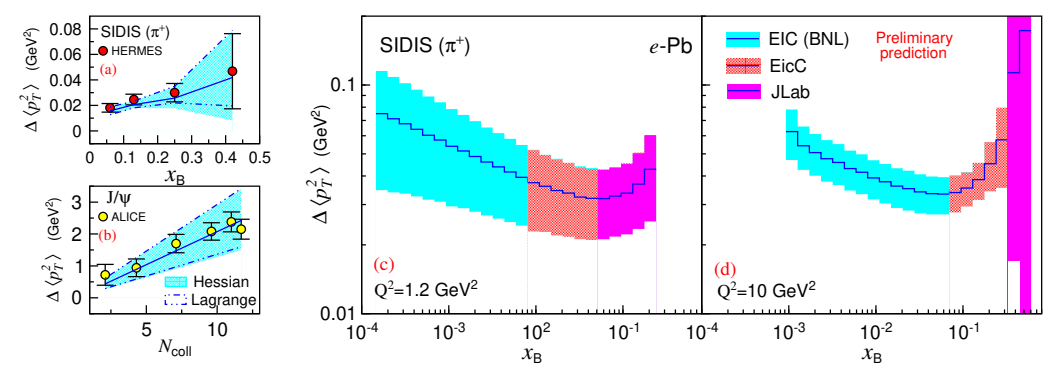

Figure 3: Panels (a-b) compare uncertainties of theoretical results estimated with Hessian matrix to those with Lagrange multiplier method. Panels (c-d) show preliminary predictions for $p_{T}$ broadening in SIDIS in future EIC experiments, with uncertianties from $\hat{q}\left(x_{B}, Q^{2}\right)$ estimated with Hessian matrix.

With the extracted $\hat{q}\left(x_{B}, Q^{2}\right)$ and error evaluation sets $S_{ \pm k}$, we can make predictions for the future EIC experiments. Some preliminary results for SIDIS at three EIC facilities covering different kinematic regions are shown in panels (c) and (d) of Fig. 3.

\section{Summary}

Quantitative evidence of the universality and non-trivial kinematic dependence of the jet transport coefficient in cold nuclear matter is presented in the first global analysis with world data. A Hessian analysis has been performed to evaluate the uncertainty of $\hat{q}$ and to make it more convenient to theoretically predict the future measurements. Preliminary predictions for future EIC experiments are also presented in this prioceeding.

Acknowledgement: This work is supported in part by NSFC of China under Project No. 11435004, by the National Science Foundation in US under Grant No. PHY-1720486, by the Science and Technology Program of Guangzhou (No. 2019050001), and by the China Postdoctoral Science Foundation under Project No. 2019M652929.

\section{References}

[1] J. Casalderrey-Solana and X. N. Wang, Phys. Rev. C 77, 024902 (2008).

[2] T. Liou, A. H. Mueller, and B. Wu, Nucl. Phys. A916, 102 (2013).

[3] Z.-B. Kang, E. Wang, X.-N. Wang, and H. Xing, Phys. Rev. Lett. 112, 102001 (2014).

[4] J.-P. Blaizot and Y. Mehtar-Tani, Nucl. Phys. A929, 202 (2014).

[5] P. Ru, Z. B. Kang, E. Wang, H. Xing and B. W. Zhang, arXiv:1907.11808.

[6] Y. Y. Zhang, G. Y. Qin and X. N. Wang, Phys. Rev. D 100, 074031 (2019).

[7] F. Arleo and C. J. Naïm, JHEP 07, 220 (2020).

[8] A. Kumar, A. Majumder and C. Shen, Phys. Rev. C 101, 034908 (2020).

[9] M. Arratia, Y. Song, F. Ringer and B. V. Jacak, Phys. Rev. C 101, 065204 (2020).

[10] J. Pumplin, D. R. Stump and W. K. Tung, Phys. Rev. D 65, 014011 (2001). 